\title{
Rh-Matched Transfusion through Molecular Typing for $\beta$-Thalassemia Patients Is Required and Feasible in Chinese
}

\author{
Chao-Peng Shao ${ }^{a}$ Cheng-Jiang Zhao ${ }^{b}$ Chang-Lin Wu ${ }^{a}$ Hua Xu ${ }^{c} \quad$ Xue-Dong Wang ${ }^{d}$ \\ Xiao-Ying $\mathrm{Wu}^{\mathrm{e}}$ Ping $\mathrm{Yi}^{\mathrm{a}}$ Xin-Tang Dang ${ }^{\mathrm{a}}$ \\ a Department of Blood Transfusion, The First Affiliated Hospital of Shenzhen University School of Medicine, The Second People's \\ Hospital of Shenzhen, Shenzhen, China; \\ bInstitute of Translational Medicine, The First Affiliated Hospital of Shenzhen University School of Medicine, The Second People's \\ Hospital of Shenzhen, Shenzhen, China; \\ ' Shanxi Blood Center, Xi'an, Shanxi, China; \\ dDepartment of Clinical Laboratory, The Fifth People's Hospital of Wuxi, The Affiliated Hospital of Nanjing Medical University, \\ Wuxi, Jiangsu, China; \\ e Shenzhen Blood Center, Shenzhen, China
}

\section{Keywords}

$\beta$-Thalassemia $\cdot$ RHCE genotyping $\cdot$ Rh-matched transfusion

\section{Summary}

Background: Molecular typing for RHCE blood group alleles has been established in many countries for patients and blood donors. In the Chinese literature nearly $80 \%$ of transfused patients with alloimmunization have antibodies specific for antigens of the Rh blood group system. We investigated if it is feasible to match packed red blood cells (RBCs) for Chinese $\beta$-thalassemia patients by RHCE genotyping. Methods: In this study, 481 patients with $\beta$-thalassemia were enrolled. They were genotyped for RHCE alleles by a simple PCR method with sequencespecific primers (PCR-SSP). Among these patients, 203 continuously received RBCs of the identical $\mathrm{Rh}$ subgroups according to the genotyping results for at least 3 months. Subsequently, their phenotypes were tested through a micro-column gel card method. For validation purposes, 400 donors were serologically typed with the same technology, of which 164 were genotyped too. Finally, the C, c, E, and e frequencies and the feasibility of the simple genotyping method were analyzed. Results: All patients showed mixed-field agglutination in the $\mathrm{Rh}$ subgroup gel cards before the same Rh subgroups in blood donors were selected for blood transfusion. The results, however, lacked mixed-field agglutination in all
203 cases after transfusion with RBC concentrates selected for the patient's C, c, E, and e antigens for at least 3 months. The genotyping results of 164 donors were all consistent with the serological results. Whole coding regions of $R H C E$ were sequenced in 7 individuals with weak $\mathrm{C}, \mathrm{E}$, or e antigens. In only one sample we observed a $1059 \mathrm{G}>\mathrm{A}$ nucleotide mutation coding for a truncated RhCE polypeptide (GenBank KT957625), in the other 6 samples no sequence variant was found. Both patients and donors were predominantly $\mathrm{CcEe}$ and CCee, with a prevalence of $55.3 \%$ and $24.9 \%$ for patients or $49.3 \%$ and $31.3 \%$ for donors, respectively. It revealed that about $80 \%$ of Chinese could receive Rh-matched RBCs easily. Conclusion: A simple RHCE genotyping technique is safe enough for Rh-matched transfusion of $\beta$-thalassemia patients in Chinese Han.

(c) 2018 S. Karger GmbH, Freiburg

\section{Introduction}

Many patients with $\beta$-thalassemia chose regular blood transfusion rather than bone marrow transplantation. Transfusion with effective iron chelation therapy has dramatically improved life expectancy of $\beta$-thalassemia patients in China in recent years. Many of them can live normally. However, 'red cell alloimmunization'

\section{KARGER}

() 2018 S. Karger GmbH, Freiburg
Chao-Peng Shao, MD

Department of Transfusion

The First Affiliated Hospital of Shenzhen University School of Medicine

The Second People's Hospital of Shenzhen

Shungang West Road No. 3002, Futian, Shenzhen 518035, China cpshaos@hotmail.com 
became a new challenge for them [1]. The incidence of alloantibodies varies in different ethnic groups, and so is the antibody specificity [2-4]. About $15-40 \%$ of $\beta$-thalassemia or other chronically transfused patients are alloimmunized according to the literature [5-7]. In China, investigations from Taiwan and Hong Kong independently reported that $10-23 \%$ of transfused $\beta$-thalassemia patients have alloantibodies $[8,9]$. According to the Chinese literature, including studies with large sample numbers, about 50-80\% of antibodies are specific for the Rh subgroup antigens $\mathrm{C}, \mathrm{c}, \mathrm{E}$, and e [10-13]. Therefore, Rh-matched transfusion is clinically important for Chinese patients, especially for those individuals who receive blood transfusions regularly [14].

In China, $\beta$-thalassemia patients are mainly located in the southern part, including Guangdong, Guangxi, Fujian, Hong Kong (near Guangdong province), and Taiwan (near Fujian province). Most of them choose therapy of regular blood transfusion in local hospitals. Unfortunately, very few studies investigated the relevance of Rh-matched transfusion in China, and according to the transfusion guidelines, red blood cell (RBC) concentrates have to be matched for $\mathrm{ABO}$ and $\mathrm{RhD}$ only. Outside China, there are many reports of Rh-matched blood transfusions in chronically transfused patients, some of them demonstrate that RHD and RHCE genotyping methods are feasible and helpful for the determination of the patient's correct Rh blood group $[15,16]$. However, some reports describe that a few patients could still develop anti-Rh antibodies, even following Rh-matched transfusions. For example, Chou et al. $[17,18]$ published in 2013 that $45 \%$ of African-American patients, who received Rh-matched RBCs according to PCR-SSP results, developed anti-Rh antibodies at the Children's Hospital of Philadelphia. The authors concluded that genetic variations caused falsepositive results in the PCR-SSP tests. In this study, we predicted the RhCcEe phenotypes with a simple RHCE genotyping technique in $\beta$-thalassemia patients in our hospital and performed transfusion with RBCs from donors with the same Rh subgroup, if available. Thus, we evaluated the feasibility of Rh-matched transfusion in Chinese clinics.

\section{Material and Methods}

\section{Subjects}

Among regular transfused $\beta$-thalassemia patients in the First Affiliated Hospital of Shenzhen University School of Medicine, 481 patients were enrolled from October 2013 to September 2017 after informed consent. We applied RHCE genotyping in these patients and selected RBCs with the same Rh subgroup type, if possible. We excluded $\mathrm{RhD}$-negative (less than $0.3 \%$ in Chinese Han population and only $1 \beta$-thalassemia patient in our hospital), autoantibody-positive patients (about 4\% who apply for washed RBCs), and patients with unidentified adverse transfusion reactions in the medical history. In addition, we included 12 patients with anti-CcEe ( 2 of anti-C, 1 anti-e, 8 anti-E, and 6 anti-c), but excluded those who had alloantibodies different from anti-C, antic, anti-E, or anti-e (more than $1 \%$ in our hospital). All enrolled patients are Han Chinese. The majority was from Guangdong, Guangxi, and Fujian provinces, others were from Sichuan, Hubei, and Hunan. There were 270 males and 211 females (1:0.78). The age distribution of the patients was as follows: 141 patients were $0-5$ years old, 181 between 6 and 10 years, 71 were $11-15$ years, 59 were 16-20 years, 25 were $21-30$ years, 3 were $31-40$ years, and 1 patient was 42 years old. Most of the patients received the first blood transfusion at the age of 7-8 months, others at 3 months or after 2 years. All patients were diagnosed with $\beta$-thalassemia by genetic tests in our hospital or in their local hospitals. Most patients came for blood transfusion 1 to 2 times per month.

\section{Controls}

400 samples of suspended leukocyte-reduced RBCs were collected, and the $\mathrm{Rh}$ subgroup phenotype was determined. Additionally, in 164 donations collected at the Shenzhen Blood Center, whole blood samples were available for DNA isolation and RHCE genotyping, which include all weak C, c, E, or e phenotype samples stored also for detailed sequencing analysis.

\section{Blood Components}

All patients were transfused with leukocyte-reduced RBCs [19], which have been filtered to remove leukocytes and centrifuged to remove plasma. RBCs contained mannitol, citric acid, sodium citrate, glucose, sodium dihydrogen phosphate, adenine, and sodium chloride (MAP storage solution) for the preservation of RBCs. All RBCs were prepared by the Shenzhen Blood Center. In most cases, patients received components from donors with the same Rh subgroups within about 10 days. If a patient was transfused in another city during this study, he or she received RBCs prepared according to the same standard operation procedure (SOP) [19].

\section{Blood Group Serology}

Serologic typing for the Rh subgroups $\mathrm{C}, \mathrm{c}, \mathrm{E}$, and e was conducted by a micro-column gel card method [20] using RhDCcEe gel cards (Changchun Boxun Biotechnology Co., Ltd., Changchun, China) or RhCeEe gel cards (Diagnostic Grifols, Parets del Vallès, Spain). The detailed SOP followed the instructions of the manufacturer. Supplied centrifuges were used.

\section{Molecular Analysis of RPHCE}

DNA was extracted from whole blood or buffy coat. RHCE genotyping was carried by Tianjin Xiupeng Biotechnology Development Co., Ltd. (Tianjin, China) using its own products (20153400740 through a sequence-specific primer PCR technique (PCR-SSP). The $R H C E^{\star} C$ primers target to the 109-bp insert in intron 2 of the $R H C E^{\star} C$ allele. $R H C E^{*} C$ primers target to exon 2 of $R H C E^{\star} c$. Finally, $R H C E^{\star} E$ and $R H C E^{\star} e$ were genotyped by testing for the allelespecific nucleotide polymorphism in exon 5. $10 \mu \mathrm{l}$ of DNA and dNTP buffer were added into PCR tubes with primers and amplified with $96^{\circ} \mathrm{C}$ for $120 \mathrm{~s}, 5$ cycles of $96^{\circ} \mathrm{C}$ for $20 \mathrm{~s}$ and $68^{\circ} \mathrm{C}$ for $60 \mathrm{~s}, 25$ cycles of $96^{\circ} \mathrm{C}$ for $20 \mathrm{~s}, 65^{\circ} \mathrm{C}$ for $50 \mathrm{~s}$ and $72^{\circ} \mathrm{C}$ for $45 \mathrm{~s}$, and $72^{\circ} \mathrm{C}$ for $120 \mathrm{~s}$ at last. In several samples the fulllength coding region of $R H C E$ was sequenced as previously described [21].

\section{Rh-Matched Transfusion}

Every Wednesday and Saturday are Rh-matched transfusion days for $\beta$-thalassemia patients in our hospital. Patients should make an appointment at least one day ahead. According to the name list and their RHCE genotyping results, the immunohematology laboratory searched the same Rh subgroup type from all available RBCs for every patient by using micro-column gel cards and then labeled the component packages with the patient's name. For some $\mathrm{Rh}$ phenotypes, we had to provide RBCs with a different Rh subgroup because RBCs with the required type were not available. Furthermore, in line with the national transfusion guidelines an antibody screening was performed before each RBC dispatch, and a wet crossmatch was performed too. During or after transfusion, the ward personnel recorded any adverse transfusion reaction in the medical record and provided the information through an adverse event reporting system.

\section{Results}

\section{Rh Subgroup Phenotyping in Patients}

We tried to phenotype and record samples from $50 \beta$ thalassemia patients before starting RBC transfusion with the same 


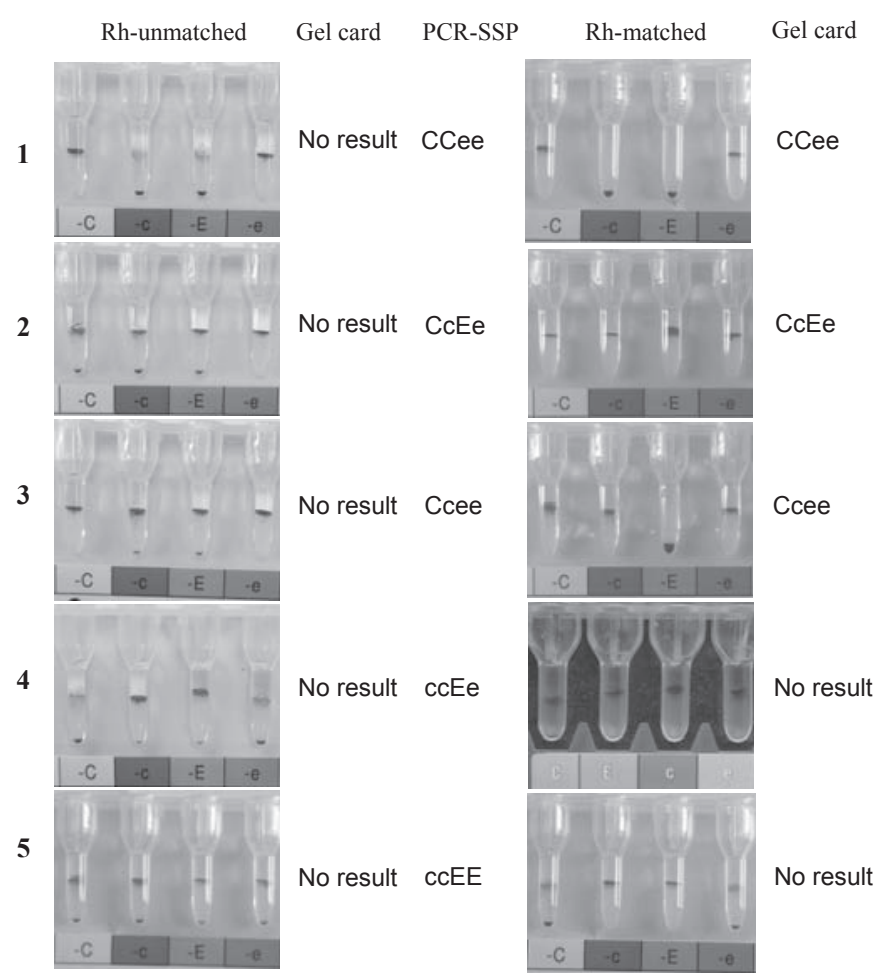

Fig. 1. RhCcEe typing before and after Rh-matched transfusions. No. 1-5 showed mixed field agglutination in the gel column in different antigen detections or in all CcEe antigen tests before Rh-matched transfusion performed for those patients. According to the genotyping results, No. 1-3 performed identical RhCcEe phenotype transfusions at least for 3 months. Those patients then had clear CcEe phenotyping results in the gel cards. In No. 4 and No. 5, C+ $\mathrm{c}+\mathrm{E}+\mathrm{e}+$ donor RBCs were transfused.

Rh subgroup. As expected, there was no patient with an unequivocal Rh subgroup phenotyping result using a micro-column gel card method. Most samples had mixed-field agglutination results in 2 or 3 gel columns or even all 4 Rh-subgroup testing columns (left column of fig. 1), which means ' $4+$ ' positive agglutination RBCs at the top of the micro-columns and ' 0 ' negative cells at the bottom of the same micro-column, simultaneously. Totally, more than $90 \%$ (47/50) samples showed mixed-field agglutination with anti-c and anti-E, whereas, less than $50 \%$ were found positive for anti-C and anti-e. These results suggested that c- and E-matched transfusion is most important for Chinese patients.

\section{Rh-Matched Transfusion}

During the study period, $481 \beta$-thalassemia patients were genotyped for RHCE by a PCR-SSP method (fig. 2). The immunohematology laboratory screened blood components according to the patient's genotyping results and issued an RBC with the same Rh subgroups through a transfusion management information system whenever possible. No feedbacks of adverse transfusion reaction were received during or after the transfusions in all patients.

Starting from June 2015, we used the transfusion management information system to search for patients who received always $\mathrm{RBCs}$ with the identical RhCE type for at least 3 consecutive

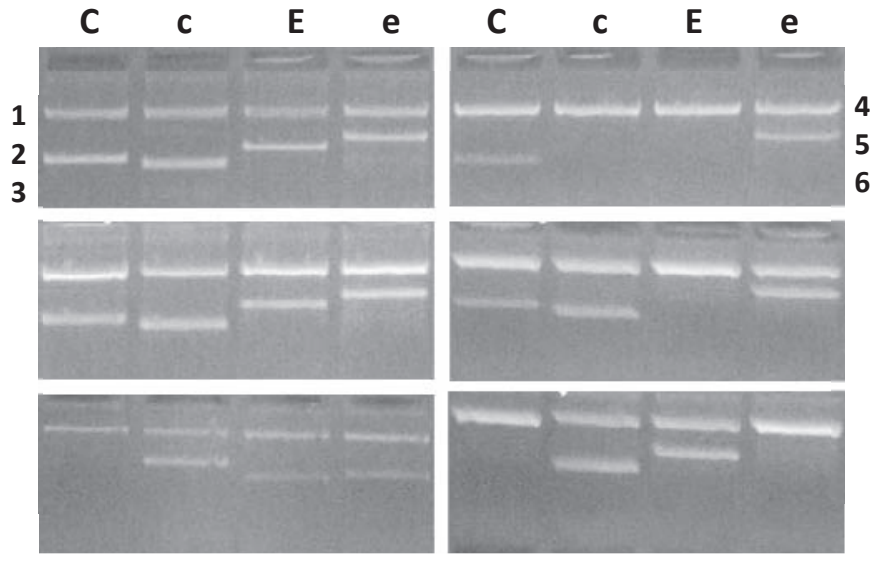

Fig. 2. RHCE genotyping by PCR-SSP. C, c, E, and e indicated RhCE antigens. No. 1 was a control sample of CcEe phenotype, No. 2-6 were patients of CcEe, ccEe, CCee, Ccee, and ccEE types.

months. Thus 203 patients identified, of whom 115 patients were found with CCee, 78 with CeEe ,and 10 with Ccee. In all these patients, we tested RhCcEe phenotypes with micro-column gel cards again. All samples showed clearly readable results. Unexpectedly, no mixed-field agglutination was observed, and no weak positive results were found. Finally, all 203 phenotyping results were concordant with the genotyping results (1,2 and 3 of fig. 1), the PCRSSP did not reveal false-positive or false-negative results. It seems that a simple DNA genotyping technology is reliable for Rhmatched RBC transfusion in clinics for Chinese.

Additionally, 5 patients with the more rare ccEe phenotype, who applied for Rh-matched transfusion, received identical Rh types or RBCs with the CcEe type because of the difficulty of finding donor blood with the identical Rh subgroups every time. The phenotyping results in the gel cards of those patients after 3 months transfusion showed mixed-field agglutination with anti-C, whereas there were exclusively clear results with anti-c, anti-E, and anti-e. This indicated obviously that the patients had previously received CcEe erythrocytes (4 of fig. 1). Moreover, 3 patients with ccEE received CcEe RBCs. Their gel card results then showed mixed-field agglutination with anti-C and anti-e, but clear results with anti-c and anti-E (5 of fig 1). These observations further prove that the simple RHCE genotyping method predicts correct $\mathrm{RhCE}$ phenotypes and is feasible for Chinese.

\section{RHCE Sequencing}

The RhCE phenotypes of 400 donor samples were investigated and meanwhile 164 of them were genotyped (fig. 2). All genotyping results were in concordance with serology, confirming the microcolumn gel card results mentioned above und further supporting the conclusion that RHCE molecular typing is a very reliable tool. Among 400 blood donors, 7 individuals with weak RhCE phenotypes were observed (table 1). The exons 1 to 10 of RHCE were amplified and sequenced in these 7 samples. One sample (No. 3, Ccee, table 1) revealed a G/A heterozygous result at nucleotide position 1059 (fig. 3), suggesting one base mutation (1059G>A) in exon 7 of 
RHCE (Genbank KT957625) which results in a stop codon at amino acid position 353. But no nucleotide variation was observed in the other 6 samples.

\section{Frequencies of RhCE Phenotypes}

Both patients and donors were predominantly CcEe and CCee phenotypes, with a prevalence of $55.3 \%$ and $24.9 \%$ in patients and $49.3 \%$ and $31.3 \%$ in donors, respectively. This indicates that more than $80 \%$ of Chinese can easily receive Rh-matched RBC transfusions (table 2). The frequencies of $\mathrm{C}$ and e antigens were $72.8 \%$ and $78.3 \%$, respectively, when the results from patients and donors were combined for calculation. Furthermore, the frequencies of $c$ and $\mathrm{E}$ antigens were $27.2 \%$ and $21.7 \%$, respectively.

\section{0}

\section{T GR AACG G CAA T G}

Fig. 3. $R H C E$ sequencing of the weak c sample. The arrow showed a G/A heterozygosity at nucleotide position 1059 in exon 7 of RHCE in the sample No. 3 of table 1.

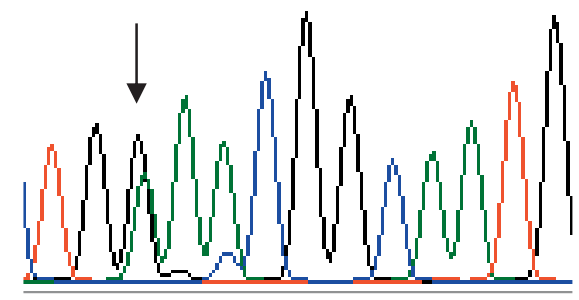

Table 1. Serological and genotyping results of $\mathrm{Rh}$ weak expression samples

\begin{tabular}{llll}
\hline No. & Gel card types & Weak positive $(\leq 2+)$ & PCR-SSP types \\
\hline 1 & CCee & weak e & CCee \\
2 & CcEe & weak c and weak E & CcEe \\
3 & Ccee & weak c & Ccee \\
4 & CcEe & weak E & CcEe \\
5 & ccEe & weak e & ccEe \\
6 & CcEe & weak e & CcEe \\
7 & ccEe & weak e & ccEe \\
\hline
\end{tabular}

\section{Discussion}

Currently, it is not applicable in chronic transfusion patients to identify RhCE phenotypes for, unless serological blood group determination is performed before initiation of transfusion therapy. Therefore, molecular techniques are increasingly applied to predict $\mathrm{RhCE}$ antigens [22]. However, because different ethnic groups have different genetic background and especially numerous variant RHCE alleles, many DNA genotyping techniques were found not reliable. Tax et al. [23] mentioned that $R H C E^{\star} C$ genotyping based on $48 \mathrm{C}$ was not reliable because of a $48 \mathrm{G}>\mathrm{C}$ mutation in the $R H C E^{*} c e$ allele (false-positive prediction of $\mathrm{C}$ ), and genotyping on intron 2 is unreliable because of r's (Cdes) alleles (false-negative prediction of C). Then, in France, Pham et al. [24] and KapplerGratias et al. [25] reported 572/806 individuals and 14.2\% African blood donors, respectively, with RhCE variants or variant RHCE alleles. Both studies suggested to transfuse patients with sickle cell disease and RhCE variants not only with phenotypically matched but also with genetically matched RBC units. In Brazil, Prisco et al. [26] reported that 421 of 80,961 blood samples were tested with atypical D typing results; among them $94.9 \%$ presented variant $R H C E^{*} c e$. Overall, many authors proposed that $R H$ variants should be considered to perform Rh-matched transfusion through molecular methods $[27,28]$. And it would be better to use a high-throughput DNA testing platform [29] or a next-generation sequencing (NGS) strategy [30] instead of a simple PCR-SSP as described in this study. In China, some Chinese papers [31-33] reported that the $48 \mathrm{G}>\mathrm{C}$ mutation in the $R H C E^{*} c e$ allele could be observed. Furthermore, Zhou et al. [31] reported in 2008 that $2 \mathrm{C}$-positive samples in $98 \mathrm{D}$-positive individuals lacked the $109 \mathrm{bp}$ insertion in intron 2. Shi et al. [32] found 1 C-positive samples in $186 \mathrm{D}$-positive Chinese Hans from southern China with a 109 bp deletion in intron 2 of RHCE. However, Wang et al. [33] identified no individual with this deletion when studying 942 samples. According to our data from 203 patients and 164 healthy donors, there is no discrepancy between RhCE phenotyping and molecular genotyping results. The

Table 2. Rates of RhCcEe phenotypes in this study and literature

\begin{tabular}{|c|c|c|c|c|c|c|c|}
\hline Phenotypes & Patients & $\%$ & Donors & $\%$ & Chinese $[34]^{*} \%$ & Caucasian $[36]^{\S} \%$ & African origin $[25]^{\S} \%$ \\
\hline CCee & 266 & 55.30 & 197 & 49.25 & 51.82 & 18.50 & 6.30 \\
\hline CcEe & 120 & 24.95 & 125 & 31.25 & 32.45 & 13.37 & 0.90 \\
\hline Ccee & 36 & 7.48 & 44 & 11.00 & 7.71 & 35.65 & 19.30 \\
\hline ccEE & 25 & 5.20 & 16 & 4.00 & 3.98 & 2.33 & 1.20 \\
\hline ccEe & 23 & 4.78 & 10 & 2.50 & 2.18 & 12.68 & 7.90 \\
\hline CCEe & 7 & 1.46 & 5 & 1.25 & 1.24 & 0.20 & 0 \\
\hline ccee & 1 & 0.21 & 1 & 0.025 & 0.39 & 17.17 & 64.20 \\
\hline CcEE & 2 & 0.042 & 1 & 0.025 & 0.21 & 0.07 & 0 \\
\hline CCEE & 1 & 0.21 & 1 & 0.025 & 0.01 & $<0.01$ & 0 \\
\hline Total & 481 & 100 & 400 & 100 & 100 & 100 & 100 \\
\hline
\end{tabular}


varying results from different studies indicate that more data should be accumulated in Chinese. However, to our limited knowledge, a simple PCR-SSP technology may be practicable or at least preliminary feasible for Rh-matched transfusion in Chinese.

We also investigated, if the frequency of different RhCE phenotypes allows us to perform Rh-matched transfusion for $\beta$-thalassemia patients regularly. Both Chinese literature [34] and our work demonstrated that CcEe and CCee phenotypes are present in more than $80 \%$ of Chinese. This implies that a general hospital, if a large amount of RBCs is used, could provide RhCEmatched RBCs for most of the $\beta$-thalassemia patients independent from the blood center. Even the local blood centers do not provide $\mathrm{Rh}$ subgroup phenotype-labelled blood components currently. If the local blood center can provide RhCE-marked RBC units, then a hospital could serve more than $97 \%$ of the patients who apply for Rh-matched transfusions, which include the common phenotypes of CCee and CcEe as well as Ccee, ccEE, ccEe, and other rare phenotypes. According to our data from 2016 published in Chinese [35], 20 alloantibodies were detected in 14 of total 354 $\beta$-thalassemia patients. Among them, $80 \%$ (16/20) were Rh-specific alloantibodies and $88 \%(14 / 16)$ Rh-specific alloantibodies were anti-c and anti-E being in agreement with the results of some recent larger studies [10-13]. Therefore, Ce-matched transfusion may be important in some nationalities [36]. But it is more important to avoid mismatch for c (in CC individuals) and mismatch for $\mathrm{E}$ (in ee individuals) in Chinese. However, identical type transfusion of all CcEe factors is the safest way for patients in need for long-term regular transfusion. According to our last data collection [35], $2 \beta$-thalassemia patients were newly detected with alloanti- bodies, one with anti-S and the other one who formerly was anti$\mathrm{Jk}^{\mathrm{a}}$ positive with anti-Ce. However, both patients never performed Rh-matched transfusion. Overall, it seems that RhCE-matched transfusion is simple and practicable in clinics in China compared to other countries such as France, Brazil, the US, or Thailand [17, 24, 27, 37, 38]. Moreover we would like to suggest that $\beta$-thalassemia patients in China should receive Rh subgroup-compatible blood from the beginning of transfusion therapy. It does not have to be donor blood with the same Rh subgroup, but it should be compatible in order to prevent alloimmunization.

Finally, 7 cases of weak c, E, and e phenotypes were found in 400 donor samples, but only 1 weak c sample was associated with a genetic variation in exon 7 of RHCE as shown by sequencing analysis. This is din contrast to the results reported by Wang et al. [33]. They detected nucleotide variations in most of the 36 cases with weak CcEe phenotypes. Thus more data accumulation is needed for understanding the molecular background of RHCE in Chinese.

\section{Acknowledgment}

The authors indebted to Prof. Dr. med. Tobias J. Legler, Department of Transfusion Medicine, University Göttingen, Germany, for his wonderful suggestions for improving the content of the paper and language improvement. We believe that we cannot finish the manuscript without his contribution.

\section{Disclosure Statement}

The authors declare no conflict of interest with regard to this paper.

\section{References}

1 Matteocci A, Pierelli L: Red blood cell alloimmunization in sickle cell disease and in thalassaemia: current status, future perspectives and potential role of molecular typing. Vox Sang 2014;3:197-208.

2 Zheng Y, Maitta RW: Alloimmunisation rates of sickle cell disease patients in the United States differ from those in other geographical regions. Transfus Med. 2016;3:225-230.

3 Allali S, Peyrard T, Amiranoff D, Cohen JF, Chalumeau M, Brousse V, de Montalembert M: Prevalence and risk factors for red blood cell alloimmunization in 175 children with sickle cell disease in a French university hospital reference centre. Br J Haematol 2017;4: 641-647.

4 Murao M, Viana MB: Risk factors for alloimmunization by patients with sickle cell disease. Braz J Med Biol Res 2005;5:675-682.

5 Kosaryan M, Mahdavi MR, Roshan P, Hojjati MT: Prevalence of alloimmunisation in patients with beta thalassaemia major. Blood Transfus 2012;3:396-397.

6 Sanz C, Nomdedeu M, Belkaid M, Martinez I, Nomdedeu B, Pereira A: Red blood cell alloimmunization in transfused patients with myelodysplastic syndrome or chronic myelomonocytic leukemia. Transfusion 2013; 4:710-715.

7 Azarkeivan A, Ansari S, Ahmadi MH, Hajibeigy B, Maghsudlu M, Nasizadeh S, Shaigan M, Toolabi A, Salahmand M: Blood transfusion and alloimmunization in patients with thalassemia: multicenter study. Pediatr Hematol Oncol 2011;6:479-485.
8 Chao YH, Wu KH, Lu JJ, Shih MC, Peng CT, Chang CW: Red blood cell alloimmunisation among Chinese patients with $\beta$-thalassaemia major in Taiwan. Blood Transfus 2013;1:71-74.

9 Cheng CK, Lee CK, Lin CK: Clinically significant red blood cell antibodies in chronically transfused patients: a survey of Chinese thalassemia major patients and literature review. Transfusion 2012;10:2220-2224.

10 Li CY, Huang F, Xiao J, Xu H, Gan XN, Yu LJ, He H: Characteristics and specificity of irregular antibodies in the blood types in 36287 patients (in Chinese). Chin J Blood Transfus 2015;4:412-414.

11 Yu TH, Yu HM, Liang HY, Xu TT, Liu TM: Rh phenotyping and irregular antibody identification in 5050 patients (in Chinese). Chin J Blood Transfus 2012;8: 756-758.

12 Huang K, Feng WM, Zhang YH, Huang YJ, Chen YK: Screening and identification of serum irregular antibodies in severe $\beta$-thalassemia children (in Chinese). Chin J Birth Health Heredity 2012;2:123-125.

13 Wang Q, Yan BZ, Yu Y: Analysis of erythrocyte blood group irregular antibody in 31766 patients (in Chinese). Chin J Blood Transfus 2011;11:966-968.

14 Dang XT, Wu CL, Peng J, Wu XY, Li MS, Zhu Y, Shao CP: A study on Rh-matched transfusions in patients with thalassemia (in Chinese). Chin J Blood Transfus 2015;7:800-803.
15 Bakanay SM, Ozturk A, Ileri T, Ince E, Yavasoglu S, Akar N, Uysal Z, Arslan O: Blood group genotyping in multi-transfused patients. Transfus Apher Sci 2013;2: 257-261.

16 Hojjati MT, Einollahi N, Nabatchian F, Pourfathollah AA, Mahdavi MR: Allele-specific oligonucleotide polymerase chain reaction for the determination of $\mathrm{Rh} \mathrm{C} / \mathrm{c}$ and $\mathrm{Rh} \mathrm{E} / \mathrm{e}$ antigens in thalassaemic patients. Blood Transfus 2011;3:301-305.

17 Chou ST, Jackson T, Vege S, Smith-Whitley K, Friedman DF, Westhoff CM: High prevalence of red blood cell alloimmunization in sickle cell disease despite transfusion from Rh-matched minority donors. Blood 2013;6:1062-1071.

18 Chou ST, Westhoff CM: The role of molecular immunohematology in sickle cell disease. Transfus Apher Sci 2011;1:73-79.

19 Ministry of Health of the People's Republic of China: The Guidelines for Clinical Blood Transfusion. Beijing, Ministry of Health of the People's Republic of China, 2000

20 Ministry of Health of the People's Republic of China: Technological Specifications for Chinese Blood Transfusion. Tianjing, Tianjin Science and Technology Press, 1997.

21 Yi P, Wu DZ, Peng J, Wu CL, Dang XT, Li JP, Zhang YZ, Xu H, Li CY, Shao CP: Establishing a sequencing method of RHCE gene coding region. Chin J Blood Transfusion 2015;7:769-772. 
22 Legler TJ, Eber SW, Lakomek M, Lynen R, Maas JH, Pekrun A, Repas-Humpe M, Schröter W, Köhler M Application of RHD and RHCE genotyping for correct blood group determination in chronically transfused patients. Transfusion 1999;8:852-855.

-23 Tax MG, van der Schoot CE, van Doorn R, DouglasBerger L, van Rhenen DJ, Maaskant-vanWijk PA: RHC and $R H c$ genotyping in different ethnic groups. Transfusion $2002 ; 5: 634-644$.

24 Pham BN, Peyrard T, Juszczak G, Beolet M, Deram G, Martin-Blanc S, Dubeaux I, Roussel M, Kappler-Gratias S, Gien D, Poupel S, Rouger P, Le Pennec PY: Analysis of RhCE variants among 806 individuals in France: considerations for transfusion safety, with emphasis on patients with sickle cell disease. Transfusion 2011;6:1249-1260.

25 Kappler-Gratias S, Auxerre C, Dubeaux I, Beolet M, Ripaux M, Le Pennec PY, Pham BN: Systematic $R H$ genotyping and variant identification in French donors of African origin. Blood Transfus 2014;12(suppl 1): 264-272.

26 Prisco Arnoni C, Guilhem Muniz J, de Paula Vendrame TA, de Medeiros Person R, Roche Moreira Latini F, Castilho L: RHCE variants inherited with altered $R H D$ alleles in Brazilian blood donors. Transfus Med 2016;4:285-290.

-27 Sippert E, Fujita CR, Machado D, Guelsin G, Gaspardi AC, Pellegrino J Jr, Gilli S, Saad SS, Castilho L: Variant $\mathrm{RH}$ alleles and $\mathrm{Rh}$ immunisation in patients with sickle cell disease. Blood Transfus 2015;1:72-77.
28 Guelsin GA, Sell AM, Castilho L, Masaki VL, Melo FC, Hashimoto MN, Higa TT, Hirle LS, Visentainer JE: Benefits of blood group genotyping in multi-transfused patients from the south of Brazil. J Clin Lab Anal 2010;5:311-316.

29 Reid ME, Halter Hipsky C, Hue-Roye K, Hoppe C: Genomic analyses of $R H$ alleles to improve transfusion therapy in patients with sickle cell disease. Blood Cells Mol Dis 2014;4:195-202.

30 Dezan MR, Ribeiro IH, Oliveira VB, Vieira JB, Gomes FC, Franco LAM, Varuzza L, Ribeiro R, Chinoca KZ, Levi JE, Krieger JE, Pereira AC, Gualandro SFM, Rocha VG, Mendrone-Junior A, Sabino EC, Dinardo CL: RHD and RHCE genotyping by next-generation sequencing is an effective strategy to identify molecular variants within sickle cell disease patients. Blood Cells Mol Dis 2017;65:8-15.

31 Zhou HY, Zhang YZ, Meng QB, Bai XH, Wang CR, Chao Q, Lan JC: Comparison between genotyping and serological phenotyping in RhCE blood group (in Chinese). Chin J Med Genet 2008,1:66-69.

32 Shi W, Li H, Zhang YZ, Li XJ, Liu CX, Silang ZR, Yu MM, Wang SH, Yan QY, Xu H, Zhou HY: Study on polymorphisms of $109 \mathrm{bp}$ insertion and 733C, G polymorphisms of RHCE gene in southern Chinese Hans and Tibetans (in Chinese). Chin J Blood Transfus 2013;10:960-964.
33 Wang ZH, Lan JC: Analysis of the correlation between the discrepance of phenotyped and genotyped with RhCE variants alleles (in Chinese). Chin J Blood Transfus 2012;11:1155-1160.

34 Yi P, Li CY, Shao CP: The research progress of RHCE gene and RhCcEe antigen (in Chinese). Chin J Blood Transfus 2014;12:1283-1287.

35 Dang XT, Wu CL, Wang XD, Li JP, Zhang YZ, Shao $\mathrm{CP}$ : Detection of serum antibodies in regularly transfused patients with Mediterranean anemia (in Chinese). Chin J Blood Transfus 2017;10:1135-1137.

36 Lewis M, Kaita H, Chown B: The inheritance of the Rh blood groups. I. Frequencies in 1,000 unrelated Caucasian families consisting of 2,000 parents and 2,806 children. Vox Sang 1971;6:500-508.

37 Gutensohn K, Müller SP, Thomann K, Stein W, Suren A, Körtge-Jung S, Schlüter G, Legler TJ: Diagnostic accuracy of noninvasive polymerase chain reaction testing for the determination of fetal rhesus $\mathrm{C}, \mathrm{c}$ and $\mathrm{E}$ status in early pregnancy. BJOG 2010;6:722-729.

38 Rujirojindaku P, Flegel WA: Applying molecular immunohaematology to regularly transfused thalassaemic patients in Thailand. Blood Transfus 2014;12:2835. 